\title{
Detecting delirium in patients with acute stroke: a systematic review of test accuracy
}

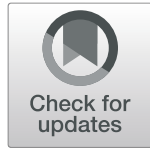

Irene Mansutti ${ }^{1}$, Luisa Saiani ${ }^{2}$ and Alvisa Palese ${ }^{3^{*}}$ (i)

\begin{abstract}
Background: Patients with acute stroke are particularly vulnerable to delirium episodes. Although delirium detection is important, no evidence-based recommendations have been established to date on how these patients should be routinely screened for delirium or which tool should be used for this purpose in this population. Therefore, the aim of this study was to identify delirium screening tools for patients with acute stroke and to summarise their accuracy.

Methods: Following the Preferred Reporting Items for Systematic Reviews and Meta-Analyses guidelines, a systematic search of Medline, CINAHL and Scopus databases was performed to include: (a) diagnostic test accuracy studies; (b) evaluating tools detecting delirium among patients with acute stroke; (c) written in English; (d) published up to September 2018. The included studies were assessed in their quality by using the Quality Assessment of Diagnostic Accuracy Studies-2.

Results: A total of four studies have been performed to date in the field with a variable quality for the methodology used and documentation of the accuracy of mainly two tools, as (1) the 4-Assessment Test for delirium (4AT), reporting a range of sensitivity from 90.2 to $100 \%$ and a specificity from 64.5 to $86 \%$; and (2) the Confusion Assessment Method-Intensive Care Unit (CAM-ICU) showing a sensitivity of 76\% (95\% Confidence of Interval [Cl] 55-91) and a specificity of 98\% (95\%Cl 93-100). Other tools have been studied as: The Abbreviated Mental Test-10, the Abbreviated Mental Test short form, the Clock Drawing Test, the Cognitive Examination derived from the National Institutes of Health Stroke Scale and the Glasgow Coma Scale. Moreover, the use of a single question —namely, 'Does this patient have cognitive issues?' as answered by the multidisciplinary team — has been subjected to a validation process.

Conclusions: To date a few primary studies have been published to test the accuracy of tools in their ability to detect post-stroke delirium; among those available, the 4AT and the CAM-ICU tools have been mostly studied. Research has just started to add evidence to the challenge of detecting and usefully assessing newly-acquired delirium among stroke patients: therefore, more studies are needed to improve the knowledge and allow a robust selection of the most useful tool to use in this population.
\end{abstract}

Keywords: Instruments, Intracerebral Haemorrhage, Ischaemic stroke, Post-stroke delirium, Tools, Sensitivity, Specificity, Systematic review

\footnotetext{
* Correspondence: alvisa.palese@uniud.it

${ }^{3}$ Department of Medical Science, University of Udine, Viale Ungheria 20,

33100 Udine, Italy

Full list of author information is available at the end of the article
}

(c) The Author(s). 2019 Open Access This article is distributed under the terms of the Creative Commons Attribution 4.0 International License (http://creativecommons.org/licenses/by/4.0/), which permits unrestricted use, distribution, and reproduction in any medium, provided you give appropriate credit to the original author(s) and the source, provide a link to the Creative Commons license, and indicate if changes were made. The Creative Commons Public Domain Dedication waiver (http://creativecommons.org/publicdomain/zero/1.0/) applies to the data made available in this article, unless otherwise stated. 


\section{Background}

Delirium is a complex syndrome characterised by disturbances in attention and awareness, associated with cognitive alterations (e.g., memory, language, perception) - which can emerge in hours or days-and tends to fluctuate in severity over time [1]. Delirium prevalence has been estimated at around 30\% in hospitalised medical patients [2], and its occurrence rate per admission has been documented to vary from 10 to $31 \%$ [3], reaching $>50 \%$ among frail elderly patients [4].

Although patients with acute stroke are particularly vulnerable to the development of this clinical complication $[5,6]$, the occurrence of delirium in this population is difficult to study because of the challenges in its detection [7, 8], while risk factors, as well as short- and long- term outcomes, have been studied more often [9], including increased functional impairments, cognitive decline, length of in-hospital stay and mortality rates [4].

Aiming at preventing delirium occurrence and at minimizing its negative consequences, several clinical guidelines have been developed to date e.g., [10, 11] which generally all recommend early detection of delirium as the basis to tailor specific interventions. Some recommendations are also available regarding patients with stroke [12], although rarely incorporated into stroke care; moreover, what tool should be used in this field is still an unresolved issue, challenging clinicians in their attempts to detect delirium early in this target population $[13,14]$.

To date only one systematic review has been published on how delirium should be screened in patients with acute stroke by summarising evidence available on sensitivity, specificity, positive and negative predictive values [13]. However, among the 20 observational studies included in the above-mentioned systematic review, none of them was aimed at evaluating tool accuracy properties (e.g., sensitivity, specificity, positive and negative predictive values) among patients with acute stroke. In addition to the Diagnostic and Statistical Manual of Mental Disorders criteria (DSM), other tools have emerged for use in practice, such as the Confusion Assessment Method (CAM), the Delirium Rating Scale (DRS), the Mini Mental State Examination (MMSE) and the Organic Brain Syndrome scale (OBS scale). However, these tools have not been validated in this specific target population [13]. Furthermore, Carin-Levy et al. [13] highlighted the heterogeneity of the methods used in delirium detection across studies, which could also explain the wide differences of documented delirium occurrence [13]. Therefore, with the purpose of updating the available review [13], as well as to summarise the evidence available on tools detecting delirium among patients with acute stroke according to the documented accuracy properties, a systematic review has been performed.

\section{Methods}

Aims

This study aimed to identify delirium screening tools for patients with acute stroke and to summarise their accuracy properties. Specifically, review aims were to (a) highlight the quantity and quality of research available on accuracy properties in the target population; (b) investigate the heterogeneity of test accuracy in the included studies; (c) identify gaps in the evidence available and determine where further research is required.

\section{Study design}

A systematic review of the literature was performed in accordance to the Preferred Reporting Items for Systematic Reviews and Meta-Analysis (PRISMA) guidelines [15]; moreover, the protocol guidance concerning test accuracy systematic reviews on problems that have a cognitive focus, was followed [16].

\section{Literature search}

Medline, the Cumulative Index to Nursing and Allied Health Literature (CINAHL) and the Scopus databases were searched up to September 2018 with the following $\mathrm{MeSH}$ terms and/or keywords combined with the Boolean operator AND/OR: (a) "Stroke", "Delirium/diagnosis", "Sensitivity and Specificity", "Neuropsychological Tests", "Validation Studies", "Diagnostic test accuracy studies" in Medline database; (b) "Stroke", "Delirium", "Validation Studies", "Neuropsychological Tests", "Clinical Assessment Tools" in CINAHL database; and (c) "Stroke", "Delirium", "Screening Tools", "Screening Tests", "Validation Studies", "Diagnostic test accuracy studies", "Clinical Assessment Tools" in Scopus database. The reference lists of the retrieved studies were also screened for additional references, and studies that published findings of retrieved study protocols were all hand-searched.

\section{Inclusion and exclusion criteria}

Eligible studies were those that satisfied the following inclusion criteria: (a) diagnostic test accuracy studies; (b) evaluating tools detecting delirium among patients with acute stroke; (c) written in English; and (d) published up to September 2018. Therefore, there were excluded articles: (a) reporting protocols regarding diagnostic test accuracy studies; (b) evaluating tools aimed at screening other cognitive issues in patients with acute stroke (e.g., dementia, cognitive decline); (c) analysing the association between post-stroke delirium and some risk factors or long-term consequences (e.g., dementia); and (d) not conducted in the acute phase of stroke, established as the first $48 \mathrm{~h}$ after the onset to the following two weeks [17]. 


\section{Study selection}

One researcher (IM) performed the literature search and two researchers (IM, AP) worked independently to evaluate study eligibility based on title and abstract screening of each study that emerged. Any differences in the evaluation regarding eligibility was discussed with a third researcher (LS). The full texts of eligible studies were then retrieved. Two researchers (IM, AP) independently evaluated each study by carefully reading the full text, and the study inclusion was decided upon joint agreement. The entire process of study inclusion is depicted in Fig. 1.

\section{Data extraction}

Two researchers (IM, AP) extracted data from each included study and populated a study-specific pro forma reporting the following information: author; year of publication; study design; country; aim(s); the tool(s) that was validated and the language of validation; the gold standard or the alternative methods (e.g., informant interviews, other tools), if any, the tool was validated against; the diagnostic practices used in the data collection process, including the timeframe and the setting(s) where the data collection was performed; who assessed the patient with the tool(s) (e.g., neurologist, student); which population was the target and which patients were involved, by extracting the inclusion and the exclusion criteria; the sampling method and the main profile (age, gender) of patients included. Moreover, all diagnostic accuracy properties assessed were also checked and extracted, such as sensitivity, specificity, positive and negative predictive values, internal consistency, accuracy, interrater reliability, likelihood ratio and the Area Under the Curve (AUC).

On a preliminary fashion, the pro forma used has been piloted by analysing two studies; then, researchers worked independently and compared the data extracted from all studies. Differences were discussed with a third researcher (LS) and full agreement was reached.

Moreover, given the wide heterogeneity regarding the terminology used by studies as: screening, detection, assessment, diagnostic tool(a), all aimed at detecting early the phenomenon of interest to allow appropriate preventive or treatment interventions, we have used "delirium detection" as an overarching term.

\section{Quality evaluation of the study included}

The included studies were evaluated in their quality by two researchers (IM, AP) using the Quality Assessment

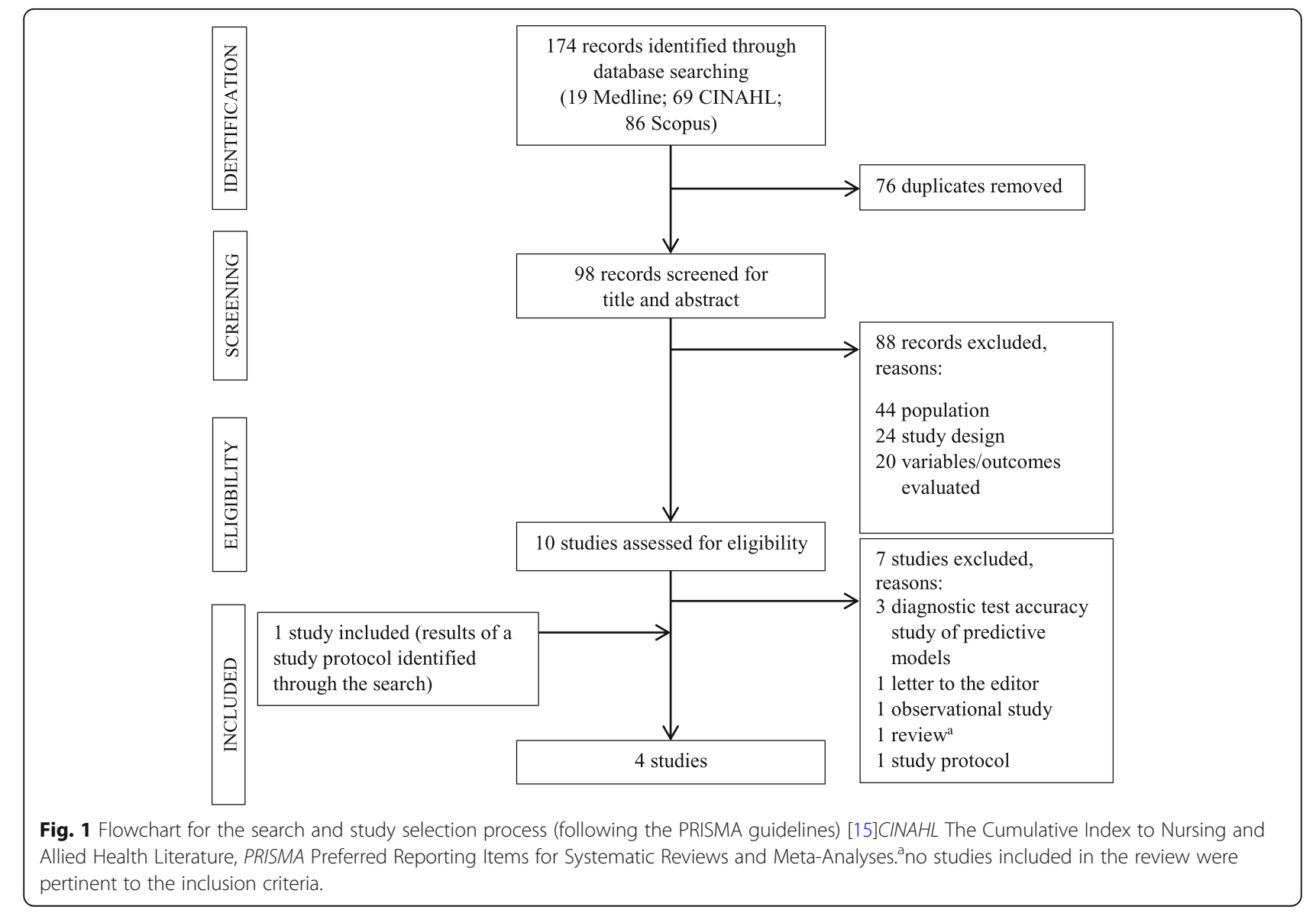


of Diagnostic Accuracy Studies-2 (QUADAS-2) [18]. Among different tools available, QUADAS-2 was chosen as precisely developed for systematic reviews of diagnostic test accuracy studies and recommended by the Agency for Healthcare Research and Quality, by the Cochrane Collaboration and by the UK National Institute for Health and Clinical Excellence [18, 19].

The tool is aimed at evaluating the "Risk of bias" that occurs if systematic flaws or limitations in (a) the patient selection, (b) the index test, (c) the reference standard used, as well as in (d) the flow and timing of the study, have threaten the findings [18]. The QUADAS-2 tool is also aimed at evaluating the "Applicability concerns", as the extent to which the primary diagnostic test accuracy is applicable to the research question addressed by the systematic review. A limited applicability can be diagnosed as follows: (a) when, as compared with the review question, the study under evaluation has been conducted in patients with different demographic and/or clinical characteristics; (b) when the index test has been applied or interpreted differently; or (c) when it has been applied with a different definition of the target condition [18]. Each QUADAS-2 tool's domain is composed of specific questions that can be answered as "yes" or "no" according to the evaluation performed by carefully reading the study. Moreover, the answer can also be "unclear" when the data available in the study under evaluation is not adequate.

\section{Results}

\section{Characteristics of the studies included}

A total of four diagnostic test accuracy studies were included in this review (Table 1), of which two $[8,21]$ had an additional observational phase and one [20] had an additional quasi-experimental phase. All studies were published from 2012 to 2017, three were conducted in Europe (Czech Republic [8]; Italy [20]; UK [22]) and one in Russia [21] and, therefore, validated in different languages.

The included studies developed diagnostic test accuracy evidence regarding the following tools: the 4-Assessment Test for delirium (4AT) [20-22], the Confusion Assessment Method for the Intensive Care Unit (CAM-ICU) [8], the Abbreviated Mental Test, in the complete and in the short version (AMT-10 and AMT-4) [22], the Clock Drawing Test (CDT) [22], the Glasgow Coma Scale (GCS) [22], and the Cognitive examination (COG4) [22]. The following gold standards/alternative tools were considered in the validation processes undertaken: three studies used the DSM criteria $[8,20,21]$ and one used the CAM [22].

Moreover, delirium detection was performed mainly by neurologists, neuropsychiatrists or physicians. Mitasova et al. [8] involved a panel of specialists who were considered "delirium experts" for the reference evaluation of delirium. However, the assessment was not always detailed if it was blinded between the tool under study and the gold standard/alternative method(s) or if the assessment was performed by one or several researchers $[20,21]$.

With regards to the timeframe, Infante et al. [20] used a single evaluation of delirium, while the remaining studies [8, 21, 22] performed several assessments; the follow-up lasted from four days after hospital admission [22] to the entire length of stay in the hospital [21].

All studies adopted a consecutive sample method using heterogeneous inclusion criteria. All included patients with both ischaemic and haemorrhagic stroke and with a certain degree of consciousness as evaluated by the clinical team or using the GCS score $>5$. However, Infante et al. [20] excluded patients with aphasia and dementia, while Mitasova et al. [8] and Kutlubaev et al. [21] also excluded patients with a history of severe head trauma, neurosurgery, brain tumour or significant chronic mental disorders/psychosis.

The study sample sizes were in general small (from 73 to 129 participants), with an age ranging from 71.3 to 79 years. Moreover, all studies were monocentric in nature and performed in hospital units, mainly in Stroke Units/Stroke centres [8, 20, 22].

\section{Quality evaluation of the studies included}

As shown in Table 2, all studies reported a low risk of concerns in the "Applicability concerns" domain. Conversely, among the "Risk of bias", the "Patient selection" and the "Reference standard" domains, major concerns have emerged, respectively due to: the exclusion of patients with specific characteristics that have potentially affected the representativeness of the sample as, for example, dementia that has been documented to raise the risk of delirium [4], and the use of a gold standard tool to detect delirium not previously validated in patients with stroke [22]. Moreover, in the same domain, with regards to the study performed by Infante et al. [20] some concerns emerged regarding the examiner's knowledge of the results obtained with the tools under evaluation when assessing delirium with the gold standard. Kutlubaev et al. [21] were instead evaluated for unclear risks in the "Index test" evaluation as it was not possible to assess if the index test findings were interpreted without knowledge of the findings of the reference standard, or not.

All studies reported a low risk of bias for the "Flow and timing" evaluation, and only Mitasova et al. [8] reported an unclear risk of bias because the appropriateness of the interval between the index test and the reference standard was not documented in the article.

\section{Accuracy properties of the tools}

As shown in Table 3, for the 4AT, the studies reported a sensitivity range of $90.2-100 \%$, a specificity range of $64.5-$ $86 \%$, a positive predictive value range of $43-86 \%$ and a 


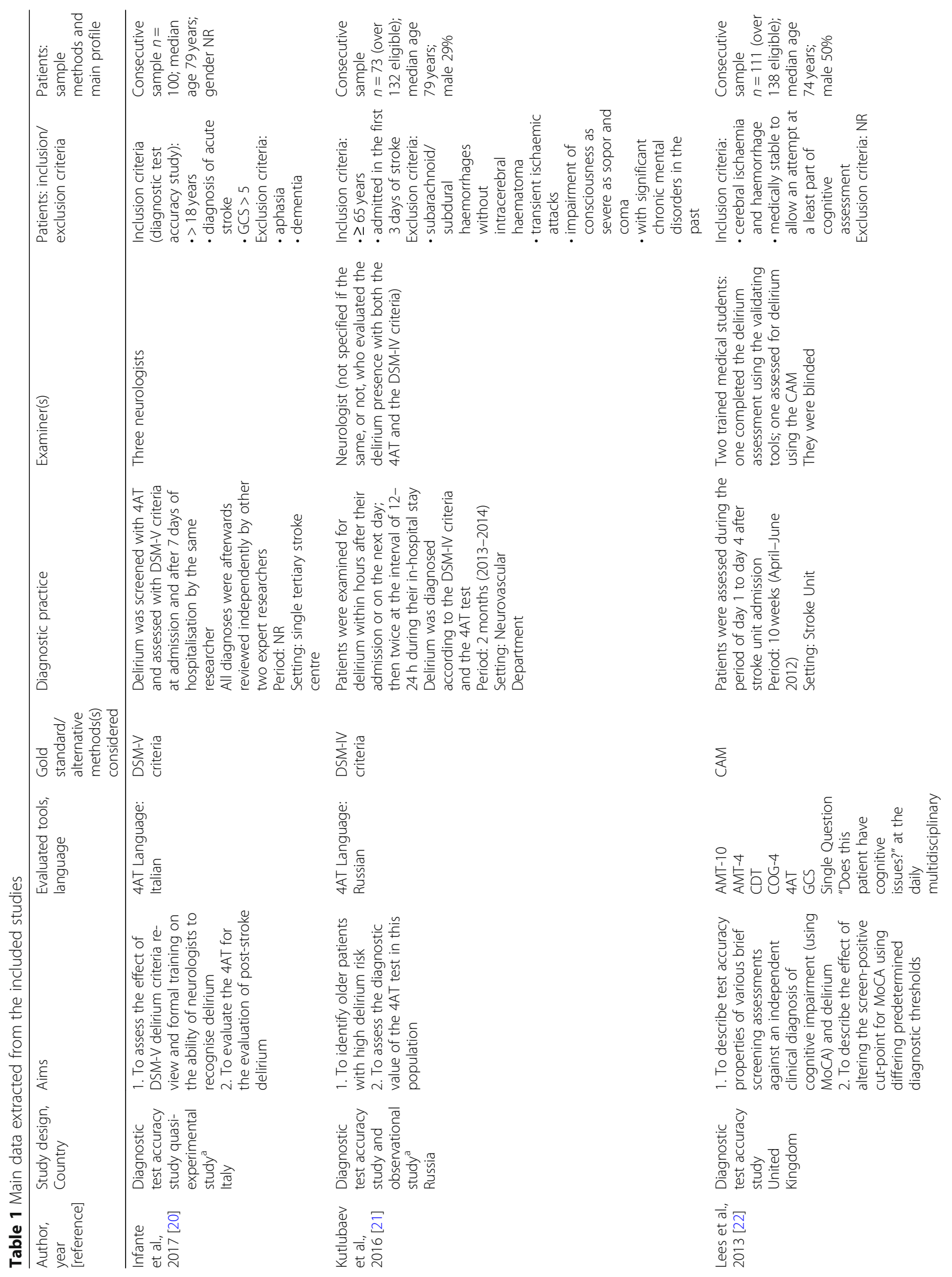




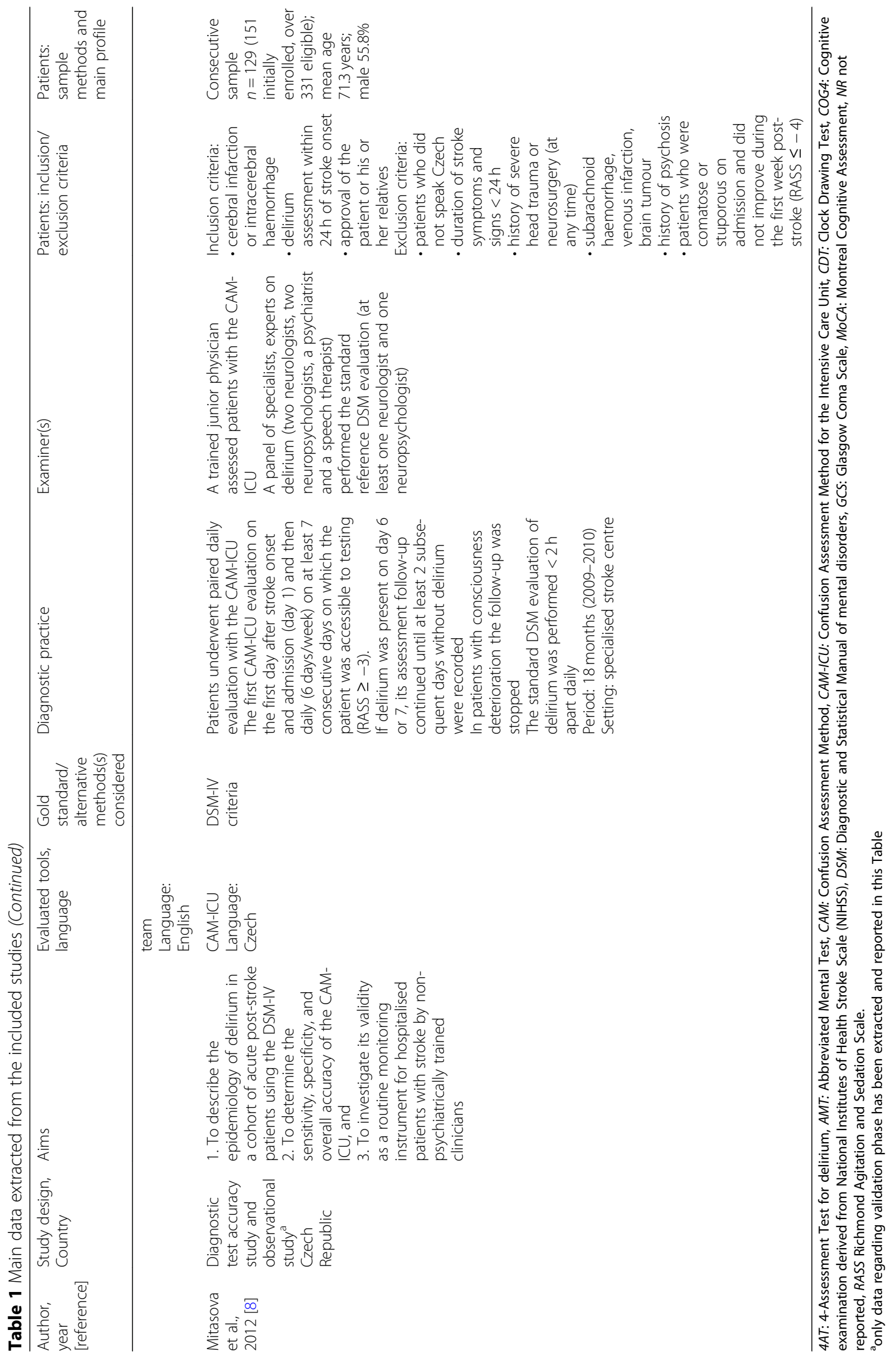




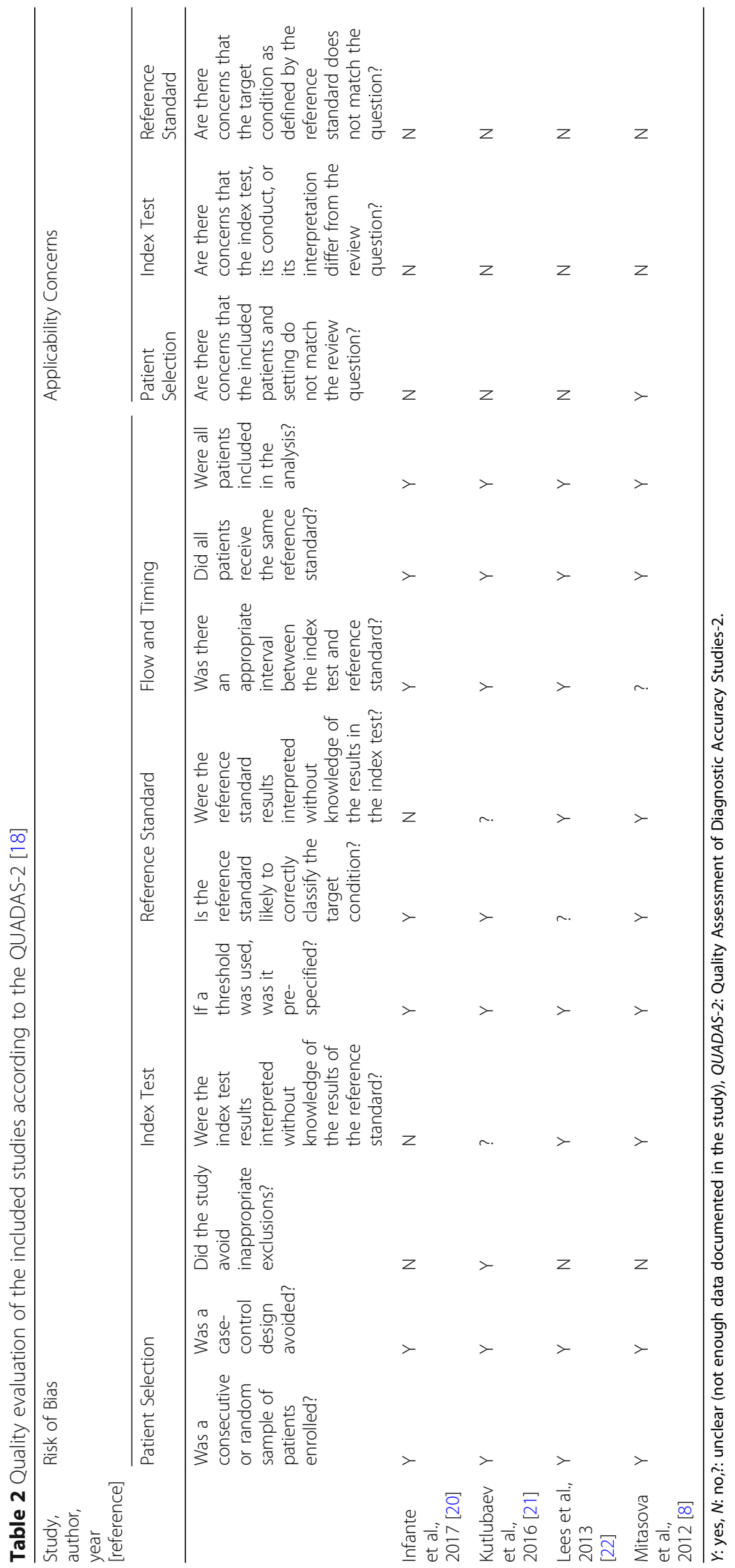




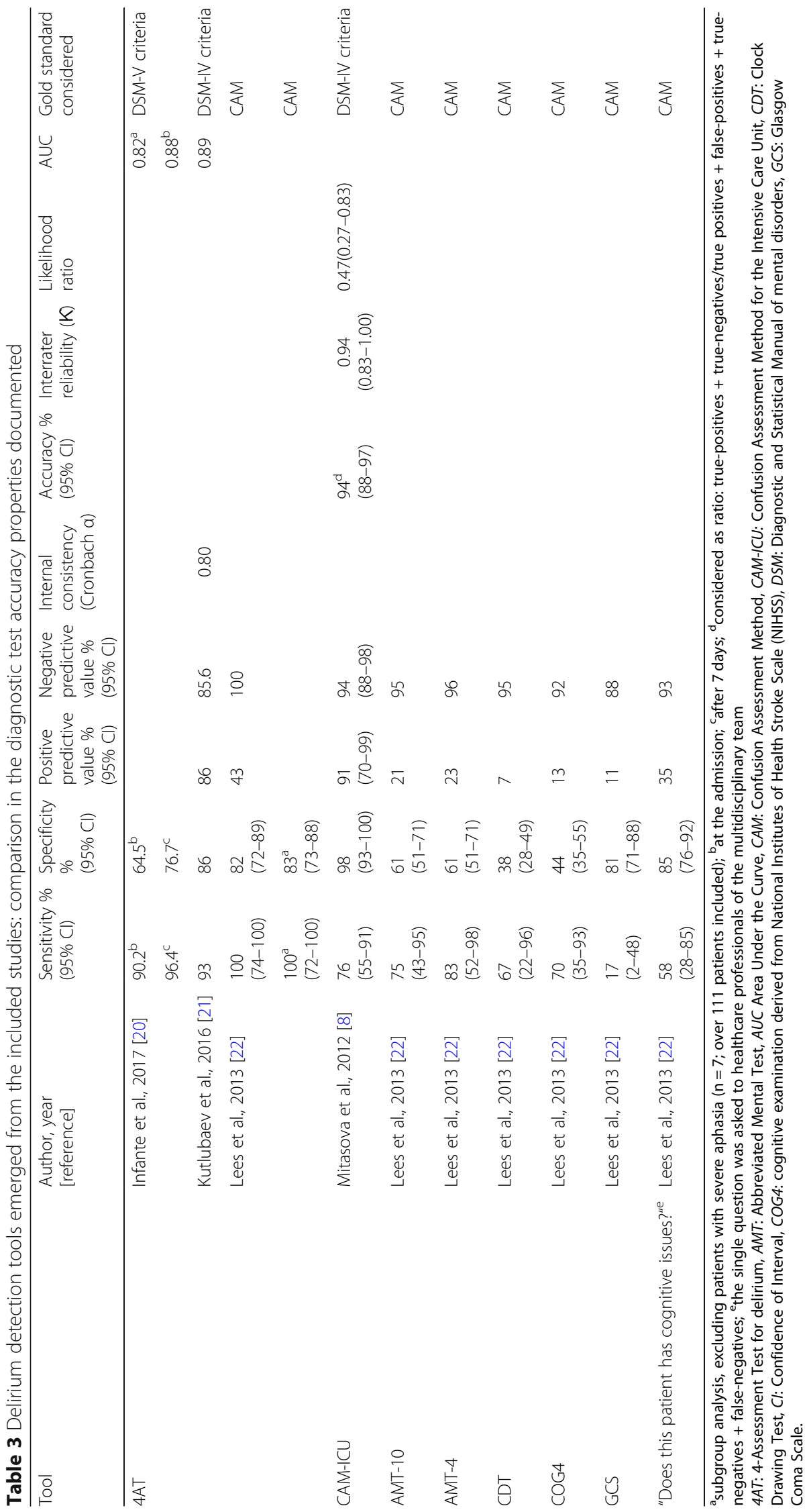


negative predictive value range of $85.6-100 \%$ [20-22]. Moreover, the AUC was evaluated only for the 4AT, ranging from 0.82 to 0.89 [20, 21].

The CAM-ICU [8] reported a sensitivity of $76 \%(95 \%$ Confidence Interval [CI] 55-91), a specificity of $98 \%$ (95\% CI 93-100), a positive predictive value of 91\% (95\% CI 70-99) and a negative predictive value of $94 \%$ (95\% CI 88-98). Additionally, Mitasova et al. [8] also estimated that the tool accuracy was $94 \%$ (95\% CI 88-97) and had an inter-rater reliability ( ) of 0.94 (95\% CI $0.83-1.00$ ), with a re-analysis of ten delirium evaluations as previously video-taped by an expert, and a likelihood ratio of $0.47(0.27-0.83)$.

Lees et al. [22] evaluated the properties of several tools, in which the AMT-10 reached a sensitivity of $75 \%$ (95\% CI 43-95) and a specificity of $61 \%$ (95\% CI 51-71), while the AMT-4 obtained a sensitivity of $83 \%$ (95\% CI $52-98)$ and a specificity of $61 \%$ (95\% CI 51-71). In the same study, the single question "Does this patient have cognitive issues?" asked to the healthcare professionals of the multidisciplinary team reached a sensitivity of $58 \%$ (95\% CI 28-85) and a specificity of $85 \%$ (95\% CI 76-92). A detailed report of the accuracy properties has been shown in Table 3 .

\section{Discussion}

We have performed a systematic review of diagnostic test accuracy studies regarding the detection of delirium among patients with acute stroke. An accurate early detection of delirium might provide opportunities to identify high risk patients, to implement evidence-based interventions designed to prevent or minimize delirium occurrence, as well as to protect patients against the consequences of delirium. Moreover, having accurate tools might also (a) reduce false positive detection, which may increase the confidence and the following use of the tool by clinicians, (b) reduce costs in the form of increased surveillance, as well as (c) reduce harm, as for example, the burden of the families called to stay at the bedside when their beloved is detected at high risk of delirium.

With regards to the first aim of this review, despite stroke patients being particularly vulnerable to delirium $[5,6]$ due to direct cerebral insult [23] and the presence of several risk factors, such as cognitive, visual and functional impairments [6] with an incidence ranging from 13 to $48 \%$ [24], only four studies to date, mainly across Europe, have investigated how to detect the delirium in these patients. Research validating delirium detection tools in patients with acute stroke has only started recently. As reported by Carin-Levy [13], no diagnostic test accuracy studies have been conducted before 2010 and all studies included in our review [8, 20-22] were published between 2012 and 2017, suggesting an increasing interest in this research field.
Concerning the quality of research studies available, some methodological limitations have emerged suggesting that further research should follow available guidelines (e.g., QUADAS-2, [18]) and assess a minimum set of accuracy properties allowing a full comparison across tools. Moreover, in available studies, tools have been used by physicians, medical students or unspecified healthcare professionals of a multidisciplinary teamsuggesting that there is a greater source of heterogeneity in their validation regarding the health care professional profile. Future studies should also involve clinical nurses given their presence at the bedside $24 / 7$ who can develop an in-depth knowledge regarding the cognitive status of the patients. Furthermore, relatives are often involved in daily practice as a reference point regarding the incurred changes in cognition as compared with the pre-stroke daily life, by detecting weak changes given their familiarity with the patient: therefore, further studies should also consider involving family members in the evaluation of the tool's accuracy. Their involvement can also increase (a) the duration of studies and their likelihood to be performed across settings (from acute to post-acute care), as well as (b) the quality of studies when family members are involved in the assessment regarding the prior clinical conditions (e.g., if patient reported, or not, delirium episodes before the acute stroke). Moreover, studies performed on this topic focused their attention on the acute phase of stroke, with assessments not lasting until the resolution of the delirium condition. Without long-term follow-ups, the strength of delirium condition detection could be uncertain and affect the accuracy properties of the tools.

A total of seven tools detecting delirium have been validated to date among patients with acute stroke. However, more than 20 tools have been developed and/ or validated in other fields, such as Intensive Care Units (e.g., CAM, CAM-ICU), surgery (e.g., CAM, Delirium Detection Score, Nurses Delirium Screening Checklist, Delirium Observation Screening Scale), emergency unit (brief-CAM, Delirium Triage Screen) and oncology/palliative care (e.g., CAM-ICU, Intensive Care Delirium Screening Checklist) [25, 26]. Several limitations have been reported among these studies [26] suggesting that in the field of neuropsychological tests researchers encounter great challenges.

The 4AT is the most validated tool in patients with acute stroke, with studies reporting good accuracy properties [20-22]. Having a tool with high sensitivity is recommended in the available literature [27] given that it can ensure an early detection of delirium which has been documented to be largely underestimated among patients with acute stroke [20]. Moreover, although a high sensitivity could lead to an overestimation of the phenomenon [20], the 4AT can allow immediate intervention at the 
onset of delirium, potentially preventing or minimizing the consequent negative outcomes. The AUC, calculated only for the 4AT [20, 21], ranged from 0.82 to 0.89 , thus, confirming the accuracy of the tool [28] — with a good internal consistency as measured with the Cronbach alpha [20] - that suggests the items included measure the same construct.

According to previous studies in the field, the $4 \mathrm{AT}$ is a rapid tool, easy to use for all healthcare professionals without specific training [25]. In addition, it has been documented to be adequate for screening patients with fluctuating levels of consciousness and drowsiness, and appears to be useful not only in hyperactive but also in hypoactive delirium [25], which has been widely reported as being underdiagnosed [14, 29]. Additionally, despite most delirium detection tools requiring a verbal response, the $4 \mathrm{AT}$ properties have been reported to not change significantly in patients with acute stroke and severe aphasia [22]. Furthermore, the 4AT has been reported to be adequate in the complex identification of delirium superimposed on dementia [25].

The CAM-ICU has been validated in patients with acute stroke only by Mitasova et al. [8]. Compared to the 4AT, the CAM-ICU demonstrated an inferior sensitivity and a higher specificity. Likewise, the 4AT has been reported to have good accuracy and a high interrater reliability across examiners, properties that cannot be compared with other tools given that these properties have only been provided for the CAM-ICU. The Likelihood Ratio data is moderate, thus, suggesting that the tool is not reliable in detecting the absence of delirium.

The CAM-ICU was originally developed for delirium detection among critically ill patients, specifically those mechanically ventilated [30,31]. Subsequently, the CAM-ICU tool has been widely validated in emergency, oncology, palliative and surgical patients [25], which are all considerably different than stroke patients. The sensitivity and specificity properties have been reported to be influenced by the health care professional's experience and training [25], thus, suggesting that the tool requires training $[25,26]$ to maximise its accuracy [25]. In contrast to the 4AT, the CAM-ICU allows a limited assessment of drowsy patients [25], with a potential risk of underdiagnosing those with hypoactive delirium.

Other delirium detection tools have been validated by Lees and colleagues [22], such as the AMT-10, the AMT-4, the CDT, the COG4 and the GCS. All reported lower psychometric properties as compared with the 4AT and the CAM-ICU, likely because these tools were not specifically designed for detecting delirium. In fact, the AMT-10 and its shortened form, AMT-4, allows a direct cognitive test of mental impairment [32, 33]. The CDT [34] does not require a verbal response from the patient, thus, it can be suitable for stroke patients [22], but it has been shown to be useful in cognition measurement-not in delirium detection [26]. Similarly, the
COG4 is derived from the National Institute of Health Stroke Scale [35] and adequately designed to evaluate the cognitive status in patients with acute stroke [35]. The issue is the same regarding the GCS [36], which has been designed to assess coma and impaired consciousness. Delirium could be a consequence of post-stroke cognitive impairments [22], but the use of the abovementioned tools in detecting delirium among these patients should be considered with caution.

To date only Lees et al. [22] have tested some properties of a single question "Does this patient have cognitive issues?", which is often used in clinical practice to detect changes in the patients' cognition by also interviewing family caregivers. Data from their study suggested that this single question has comparable specificity to other structured tools (e.g., 4AT, CAM-ICU) and superior sensitivity with regards to the CGS.

A further consideration is needed regarding the gold standard or the alternative measures criteria used across studies. Infante et al. [20], Kutlubaev et al. [21] and Mitasova et al. [8] considered the DSM-IV criteria as a point of reference for the 4AT and the CAM-ICU, respectively. The DSM-IV criteria could be considered as a gold standard, but need to be performed by experts in the field, such as neurologists or psychiatrists, and requires special training [21]. Alternatively, Lees and colleagues [22] assessed the 4AT properties against the CAM, although this tool has not been validated in delirium detection among patients with acute stroke.

Therefore, more studies are encouraged in this field to establish the accuracy of the tools detecting delirium among patients with acute stroke; alongside, these tools should be considered inside of the widely clinical examination of the patients [37].

\section{Strengths and limitations}

This systematic review offers an overview of the delirium detection tools available in the literature regarding patients with acute stroke, in which the assessment could be a challenge due to specific neurological symptoms (e.g. aphasia). Accuracy properties of different tools have been analysed, as well as the methodological quality of the studies documenting these properties. However, this review is affected by several limitations. Firstly, we did not register and publish the review research protocol in a public access repository. Moreover, only Medline, CINAHL, and Scopus databases were searched and only studies published in English and peer reviewed were included, thus introducing a potential source of selection bias. Authors were not contacted to obtain missing or incomplete data that was not reported in the included studies due, for example, to word restrictions imposed by journal guidelines, and this could have introduced 
information bias and affect the completeness of the quality evaluation as performed with the QUADAS-2 [18].

Some accuracy properties were heterogeneously reported across studies thus threatening a full comparison of the findings: for example, $95 \% \mathrm{CI}$ has been not reported in Kutlubaev et al. (2016) [18] or in Infante et al. (2017) [20]; moreover, sensitivity and specificity have all been reported while positive and negative predictive values were not. Attempts were performed to calculate the missing metrics, but no reliable findings were obtained due to some missing data in the studies: this strengthens the need to further adopt guidelines [18] in reporting test accuracy studies regarding delirium.

\section{Conclusions}

The detection of delirium in stroke is complex, given the challenges of (a) the high occurrence of sensory, perceptual and communication deficits with stroke, (b) the differentiation of delirium from more permanent stroke-related acquired cognitive impairments, and (c) the pre-existing cognitive impairments, given that vascular disease and stroke are risk factors for both dementia and further stroke. However, having instruments supporting the clinical judgment is crucial to personalise pharmacological and non-pharmacological interventions [38, 39] preventing and/or managing this clinical condition and its negative consequences.

Despite its relevance, to date few primary studies have been published to test accuracy properties of tools in their ability to detect post-stroke delirium; among those available, the 4AT and the CAM-ICU tools have been mostly studied. However, the small number of studies retrieved have documented different measures thus preventing comparisons across tools and suggesting that research has just started to add evidence to the challenge of detecting and usefully assessing newly-acquired delirium in stroke disease. Furthermore, without long-term follow-ups at least until the resolution of the delirium, it is uncertain how robust the diagnosis of delirium can be. Therefore, further studies methodologically sound and reporting a minimum data set of metrics in order to ensure comparison across studies, are strongly recommended. Moreover, alongside the importance of simple tools in the detection of newly-acquired delirium, a full consideration of the use of structured informant histories from either family or nurses and other care staff should be considered.

\section{Abbreviations}

4AT: 4-Assessment Test for delirium; AMT: Abbreviated Mental Test; AUC: Area Under the Curve; CAM: Confusion Assessment Method; CAMICU: Confusion Assessment Method for the Intensive Care Unit; CDT: Clock Drawing Test; Cl: Confidence Interval; CINAHL: The Cumulative Index to Nursing and Allied Health Literature; COG4: Cognitive examination derived from National Institutes of Health Stroke Scale (NIHSS); DSM: Diagnostic and Statistical Manual of mental disorders; GCS: Glasgow Coma Scale; MoCA: Montreal Cognitive Assessment; N: No; NR: Not reported;
PRISMA: Preferred Reporting Items for Systematic Reviews and MetaAnalyses; QUADAS-2: Quality Assessment of Diagnostic Accuracy Studies-2; RASS: Richmond Agitation and Sedation Scale; Y: Yes? Unclear (not enough data)

\section{Acknowledgements}

Not applicable.

\section{Authors' contributions}

IM performed the literature search; evaluated study eligibility by examining the titles and abstracts of the studies found in the systematic literature; evaluated the inclusion of each study by reading the full-text; evaluated the study quality and extracted the data. AP evaluated study eligibility by examining the titles and abstracts of the studies found in the systematic literature; evaluated the inclusion of each study by reading the full-text; evaluated the study quality and extracted the data. LS discussed any difference that has emerged from the independent study evaluation and data extraction of IM and AP. IM, LS and AP contributed in writing the manuscript. All authors read and approved the final manuscript.

\section{Funding}

This research received no specific grant from any funding agency in the public, commercial, or not-for-profit sectors.

Availability of data and materials

The datasets used and/or analyzed during the current study are available from the corresponding author on reasonable request.

Ethics approval and consent to participate

Ethical approval is not required for this review.

Consent for publication

Not applicable.

\section{Competing interests}

The authors declare that they have no competing interests.

\section{Author details}

${ }^{1}$ Department of Biomedicine and Prevention, University of Tor Vergata, Rome, Italy. ${ }^{2}$ Department of Diagnostics and Public Health, University of Verona, Verona, Italy. ${ }^{3}$ Department of Medical Science, University of Udine, Viale Ungheria 20, 33100 Udine, Italy.

Received: 17 August 2019 Accepted: 27 November 2019

Published online: 02 December 2019

References

1. American Psychiatric Association. Diagnostic and statistical manual of mental disorders (DSM-5). 5th ed. Virginia: American Psychiatric Association Publishing: 2013.

2. Marcantonio ER. Delirium in Hospitalized Older Adults. N Engl J Med. 2017; 377(15):1456-66.

3. Ryan DJ, O'Regan NA, Caoimh RÓ, Clare J, O'Connor M, Leonard M, McFarland J, Tighe S, O'Sullivan K, Trzepacz PT, Meagher D, Timmons S. Delirium in an adult acute hospital population: predictors, prevalence and detection. BMJ Open. 2013;3(1)

4. Inouye SK, Westendorp RG, Saczynski JS. Delirium in elderly people. Lancet. 2014;383(9920):911-22

5. Oldenbeuving AW, de Kort PL, Jansen BP, Algra A, Kappelle $L$, Roks G. Delirium in the acute phase after stroke: incidence, risk factors, and outcome. Neurology. 2011; 15:76(11):993-9.

6. Makin SD, Wardlaw J. Predicting delirium after a stroke. J Neurol Neurosurg Psychiatry. 2014;85(4):357.

7. Miu DK, Yeung JC. Incidence of post-stroke delirium and 1-year outcome. Geriatr Gerontol Int. 2013;13(1):123-9.

8. Mitasova A, Kostalova M, Bednarik J, Michalcakova R, Kasparek T, Balabanova P, Dusek L, Vohanka S, Ely EW. Poststroke delirium incidence and outcomes: validation of the confusion assessment method for the intensive care unit (CAM-ICU). Crit Care Med. 2012;40(2):484-90. 
9. Mansutti I, Saiani L, Palese A. Delirium in patients with ischaemic and haemorrhagic stroke: findings from a scoping review. Eur J Cardiovasc Nurs. 2019;18(6):435-48

10. NICE. Delirium: prevention, diagnosis and management. Clinical guideline; 2010. Available on: nice.org.uk/guidance/cg103. Accessed 11 Nov 2019.

11. Barr J, Fraser GL, Puntillo K, Ely EW, Gélinas C, Dasta JF, Davidson JE, Devlin JW, Kress JP, Joffe AM, Coursin DB, Herr DL, Tung A, Robinson BR, Fontaine DK, Ramsay MA, Riker RR, Sessler CN, Pun B, Skrobik Y. Jaeschke R; American College of Critical Care Medicine. Clinical practice guidelines for the management of pain, agitation, and delirium in adult patients in the intensive care unit. Crit Care Med. 2013 Jan;41(1):263-306.

12. Quinn TJ, Elliott E, Langhorne P. Cognitive and mood assessment tools for use in stroke. Stroke. 2018:49(2):483-90.

13. Carin-Levy G, Mead GE, Nicol K, Rush R, van Wjick F. Delirium in acute stroke: screening tools, incidence rates and predictors: a systematic review. J Neurol. 2012;259(8):1590-9.

14. Mc Manus J, Pathansali R, Stewart R, Macdonald A, Jackson S. Delirium poststroke. Age Ageing. 2007;36(6):613-8.

15. Moher D, Liberati A, Tetzlaff J, Altman DG, PRISMA Group. Preferred reporting items for systematic reviews and meta-analyses: the PRISMA statement. BMJ. 2009:21(339):b2535.

16. Davis DH, Creavin ST, Noel-Storr A, Quinn TJ, Smailagic N, Hyde C, Brayne C, McShane R, Cullum S. Neuropsychological tests for the diagnosis of Alzheimer's disease dementia and other dementias: a generic protocol for cross-sectional and delayed-verification studies. Cochrane Database Syst Rev. 2013;3.

17. NICE. Stroke and transient ischaemic attack in over 16s: diagnosis and initial management. Clinical guideline; 2008. Available on: nice.org.uk/guidance/ cg68. Accessed 20 Nov 2019

18. Whiting PF, Rutjes AW, Westwood ME, Mallett S, Deeks JJ, Reitsma JB, Leeflang MM, Sterne JA. Bossuyt PM; QUADAS-2 group. QUADAS-2: a revised tool for the quality assessment of diagnostic accuracy studies. Ann Intern Med. 2011;155(8):529-36.

19. Reitsma JB, Rutjes AWS, Whiting P, Vlassov W, Leeflang MMG, Deeks JJ. Assessing methodological quality. In: Deeks JJ, Bossuyt PM, Gatsonis C (editors), Cochrane Handbook for Systematic Reviews of Diagnostic Test Accuracy Version 1.0.0. The Cochrane Collaboration, 2009. Available from: http://srdta.cochrane.org/.

20. Infante MT, Pardini M, Balestrino M, Finocchi C, Malfatto L, Bellelli G, Mancardi GL, Gandolfo C, Serrati C. Delirium in the acute phase after stroke: comparison between methods of detection. Neurol Sci. 2017 Jun;38(6):1101-4.

21. Kutlubaev MA, Bikbulatova LF, Akhmadeeva LR. Early diagnosis of delirium in elderly patients with acute stroke. Adv Gerontol. 2016;6(1):60-6.

22. Lees R, Corbet S, Johnston C, Moffitt E, Shaw G, Quinn TJ. Test accuracy of short screening tests for diagnosis of delirium or cognitive impairment in an acute stroke unit setting. Stroke. 2013;44(11):3078-83.

23. Ferro JM, Caeiro L, Verdelho A. Delirium in acute stroke. Curr Opin Neurol. 2002;15(1):51-5.

24. Shi Q, Presutti $R$, Selchen D, Saposnik G. Delirium in acute stroke: a systematic review and meta-analysis. Stroke. 2012;43(3):645-9.

25. De J, Wand AP. Delirium screening: a systematic review of delirium screening tools in hospitalized patients. Gerontologist. 2015:55(6):1079-99.

26. Adamis D, Sharma N, Whelan PJ, Macdonald AJ. Delirium scales: a review of current evidence. Aging Ment Health. 2010;14(5):543-55.

27. Takwoingi Y, Quinn TJ. Review of diagnostic test accuracy (DTA) studies in older people. Age Ageing. 2018;47(3):349-55.

28. Swets JA. Measuring the accuracy of diagnostic systems. Science. 1988; 240(4857):1285-93

29. Hosker C, Ward D. Hypoactive delirium. BMJ. 2017;357:j2047.

30. Ely EW, Inouye SK, Bernard GR, Gordon S, Francis J, May L, Truman B, Speroff T, Gautam S, Margolin R, Hart RP, Dittus R. Delirium in mechanically ventilated patients: validity and reliability of the confusion assessment method for the intensive care unit (CAM-ICU). JAMA. 2001;286(21):2703-10.

31. Ely EW, Margolin R, Francis J, May L, Truman B, Dittus R, Speroff T, Gautam S, Bernard GR, Inouye SK. Evaluation of delirium in critically ill patients: validation of the confusion assessment method for the intensive care unit (CAM-ICU). Crit Care Med. 2001;29(7):1370-9.

32. Hodkinson HM. Evaluation of a mental test score for assessment of mental impairment in the elderly. Age Ageing. 1972;1:233-8.

33. Schofield I, Stott DJ, Tolson D, McFadyen A, Monaghan J, Nelson D. Screening for cognitive impairment using the 4-item abbreviated mental test. Eur J Emerg Med. 2010;6:340-2.
34. Richardson HE, Glass JN. A comparison of scoring protocols on the clock drawing test in relation to ease of use, diagnostic group, and correlations with mini-mental state examination. J Am Geriatr Soc. 2002;50:169-73.

35. Cumming TB, Blomstrand C, Bernhardt J, Linden T. The NIH stroke scale can establish cognitive function after stroke. Cerebrovasc Dis. 2010;30:7-14.

36. Teasdale G, Jennett B. Assessment of coma and impaired consciousness. A practical scale. Lancet. 1974;2:81-4.

37. Patel MB, Bednarik J, Lee $P$, Shehabi Y, Salluh Jl, Slooter AJ, Klein KE, Skrobik Y, Morandi A, Spronk PE, Naidech AM, Pun BT, Bozza FA, Marra A, John S, Pandharipande PP, Ely EW. Delirium monitoring in Neurocritically ill patients: a systematic review. Crit Care Med. 2018 Nov:46(11):1832-41.

38. Siddiqi N, Harrison JK, Clegg A, Teale EA, Young J, Taylor J, Simpkins SA. Interventions for preventing delirium in hospitalised non-ICU patients. Cochrane Database Syst Rev. 2016;11:CD005563.

39. Burry L, Mehta S, Perreault MM, Luxenberg JS, Siddiqi N, Hutton B Fergusson DA, Bell C, Rose L. Antipsychotics for treatment of delirium in hospitalised non-ICU patients. Cochrane Database Syst Rev. 2018;18(6): CD005594.

\section{Publisher's Note}

Springer Nature remains neutral with regard to jurisdictional claims in published maps and institutional affiliations.
Ready to submit your research? Choose BMC and benefit from:

- fast, convenient online submission

- thorough peer review by experienced researchers in your field

- rapid publication on acceptance

- support for research data, including large and complex data types

- gold Open Access which fosters wider collaboration and increased citations

- maximum visibility for your research: over $100 \mathrm{M}$ website views per year

At $\mathrm{BMC}$, research is always in progress.

Learn more biomedcentral.com/submissions 